Supporting Information

\title{
High-Yield Production of Boron Nitride Nanosheet and Its Uses as a Catalyst Support for Hydrogenation of Nitroaromatics
}

Wenliang Sun, ${ }^{\S}$ Yuan Meng, ${ }^{\S}$ Qinrui Fu, Fei Wang, Guojie Wang, Wenhua Gao, Xiaochun Huang, ${ }^{*}$ and Fushen $\mathrm{Lu}^{*}$

Department of Chemistry, Shantou University, Guangdong 515063, P. R. China.

"To whom correspondence should be addressed: xchuang@stu.edu.cn and fslu@stu.edu.cn.

${ }^{\S}$ W.L.S. and Y.M. contributed equally to this work. 


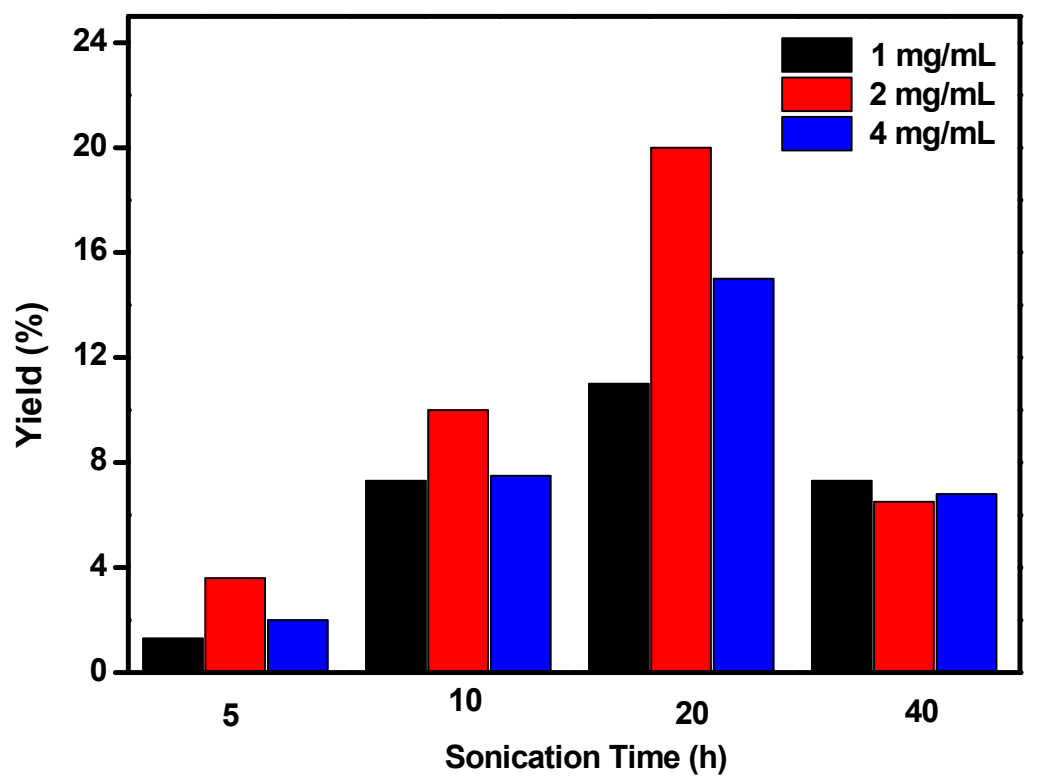

Figure S1 The influences of sonication time and original concentration of h-BN on BNNSs yield. 

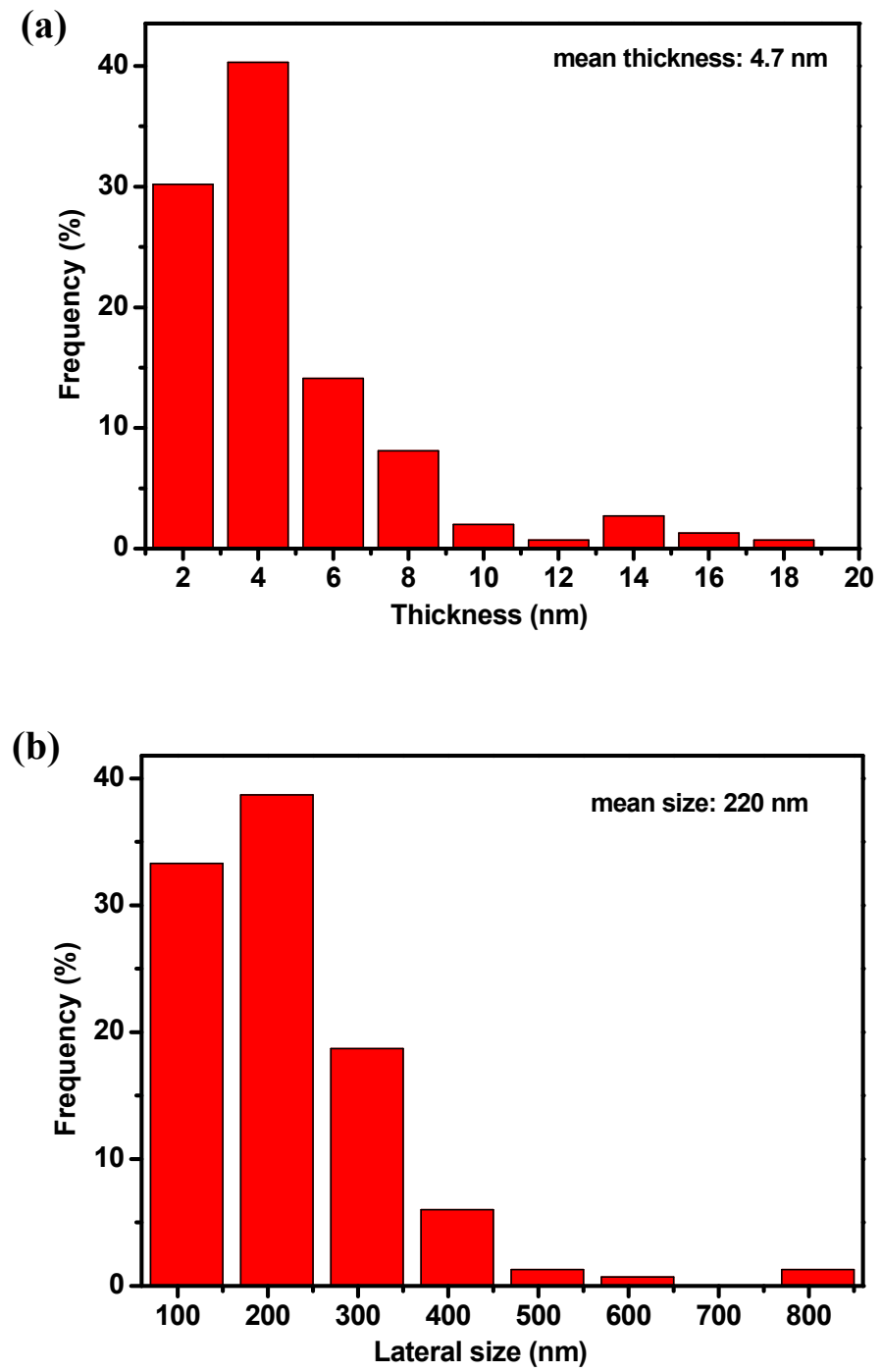

Figure S2 The distribution of thickness (a) and lateral size (b) of BNNSs determined by measuring 150 sheets in several AFM images. 

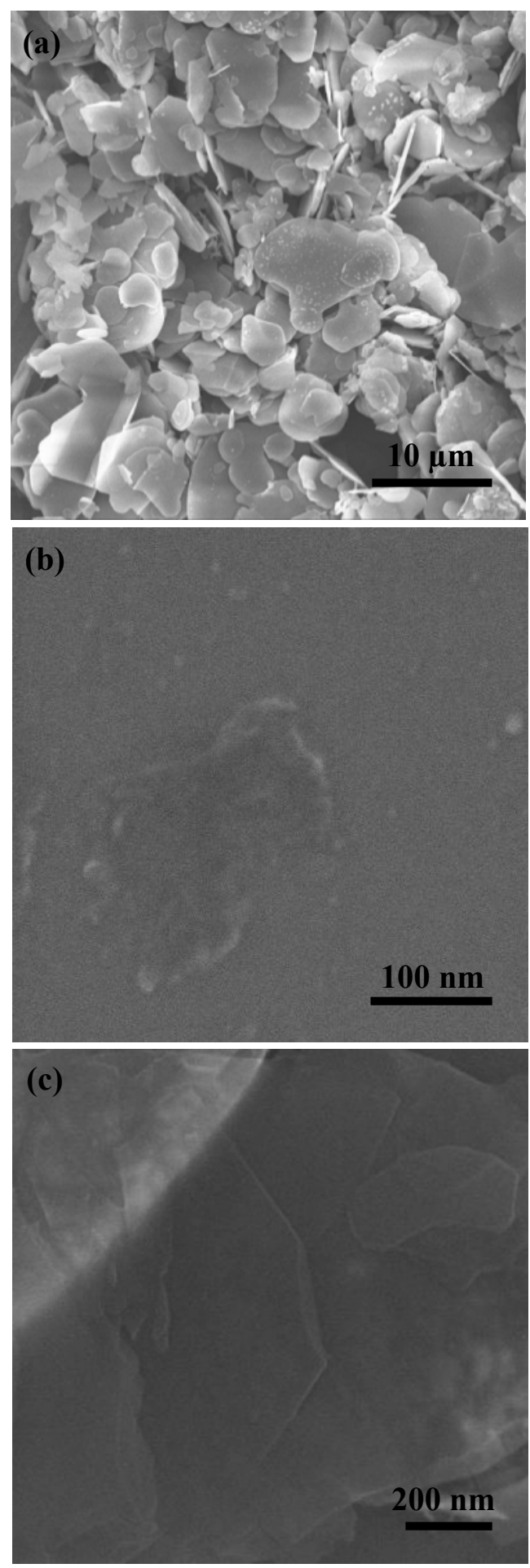

Figure S3 SEM images of the as-supplied h-BN powder (a) and BNNSs (b-c). 


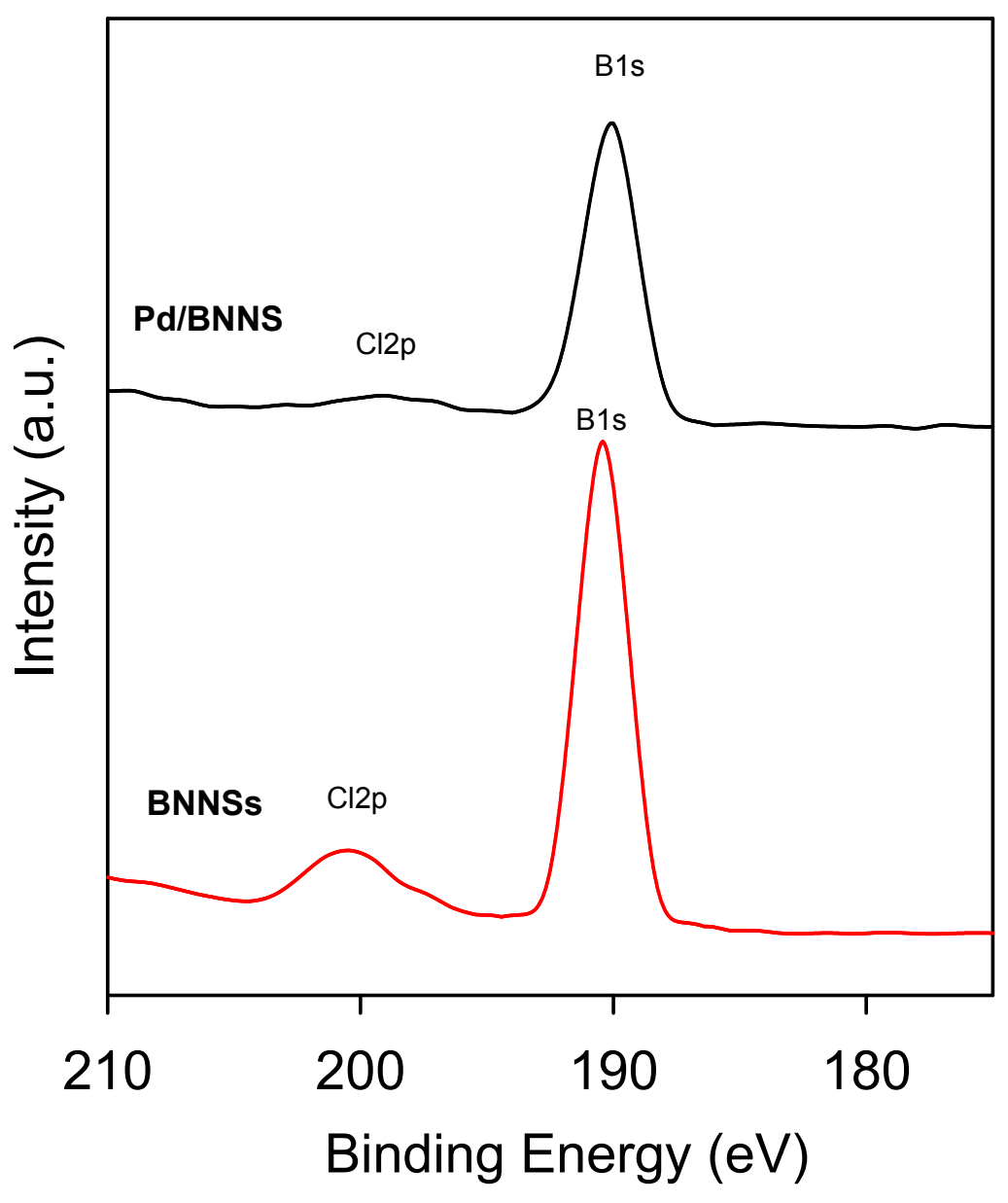

Figure S4 Expanded XPS spectra of Pd/BNNS and BNNSs showing the Cl2p and B1s peaks. 


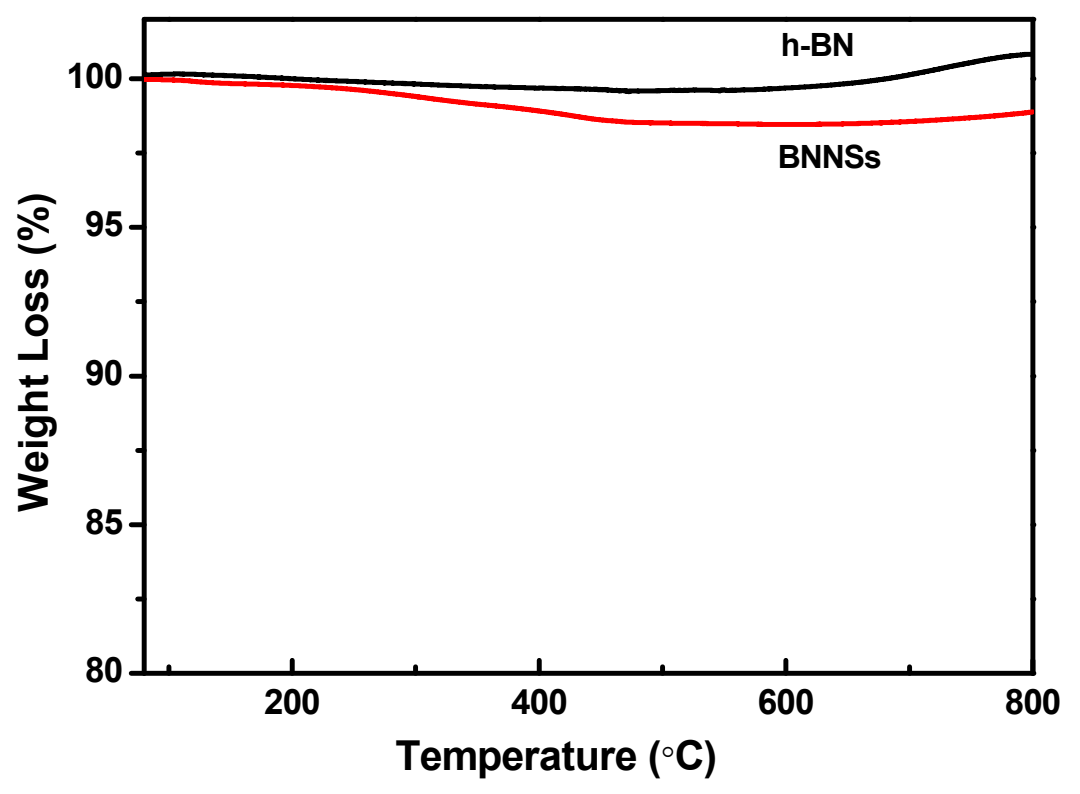

Figure S5 The TGA curves of pristine h-BN and BNNSs. 


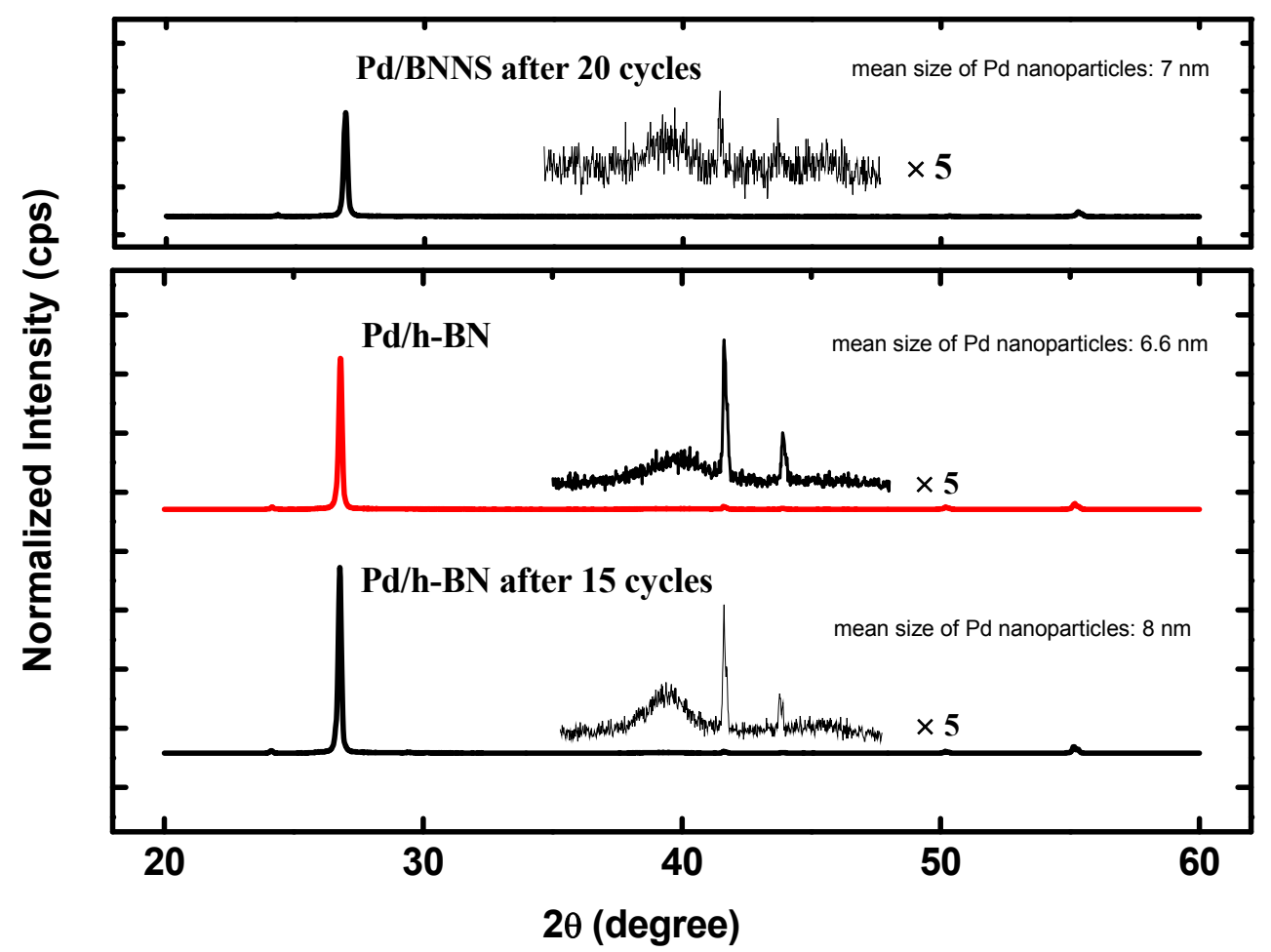

Figure S6 The XRD patterns of Pd/BNNS after 20 cycles (a), and $\mathrm{Pd} / \mathrm{h}-\mathrm{BN}$ before (b) and after 15 cycles (c). Inset: enlarged patterns in the region of 35-48 degree (Note: sizes of Pd nanoparticles were calculated with Debye-Scherrer equation). 
Table S1. Catalytic performance of different supported-Pd Catalysts for Hydrogenation of Nitrobenzene.

\begin{tabular}{|c|c|c|c|c|c|c|c|c|}
\hline Catalyst & Solvent & $\begin{array}{c}\mathrm{Pd} / \text { Substrate } \\
(\mathrm{mol} / \mathrm{mol})\end{array}$ & $\begin{array}{c}\text { Temperature } \\
(\mathrm{K})\end{array}$ & $\begin{array}{c}\text { Pressure } \\
\text { (atm) }\end{array}$ & $\begin{array}{l}\text { Time } \\
\text { (h) }\end{array}$ & $\begin{array}{c}\text { Yield } \\
(\%)\end{array}$ & $\begin{array}{c}\text { Rate } \\
(\mathrm{mol} / \mathrm{g} \cdot \mathrm{h})\end{array}$ & Reference \\
\hline $\mathrm{Pd} / \mathrm{BNNS}$ & $\mathrm{EtOH}$ & $1.27 \times 10^{-3}$ & 303 & 1 & 0.75 & 100 & 9.9 & This work \\
\hline $\mathrm{Pd} / \mathrm{C}$ & $\mathrm{EtOH}$ & $1.27 \times 10^{-3}$ & 303 & 1 & 1 & 100 & 7.4 & Commercial \\
\hline $\mathrm{SiO}_{2}-\mathrm{ILs}\left[\mathrm{PF}_{6}\right] \mathrm{Pd}^{0}$ & $\mathrm{EtOH}$ & $2.86 \times 10^{-4}$ & r. t. & 1 & 8.5 & 100 & 4.7 & {$[1]$} \\
\hline $\mathrm{Pd} / \mathrm{PEG}^{\mathrm{b}}$ & $\mathrm{EtOH}$ & $3.52 \times 10^{-2}$ & r. t. & 1 & 3 & 100 & 0.1 & {$[\underline{2}]$} \\
\hline $\mathrm{Fe}_{3} \mathrm{O}_{4}-\mathrm{NH}_{2}-\mathrm{Pd}^{\mathrm{c}}$ & $\mathrm{EtOH}$ & $1.58 \times 10^{-2}$ & r. t. & 1 & 0.75 & $>99$ & 0.8 & {$[\underline{3}]$} \\
\hline $\mathrm{Pd} / \mathrm{SiO}_{2}{ }^{\mathrm{d}}$ & THF & $9.87 \times 10^{-3}$ & 373 & 30 & 1 & 74 & 0.7 & {$[\underline{4}]$} \\
\hline \multicolumn{9}{|c|}{${ }^{\mathrm{a}} \mathrm{SiO}_{2}-\mathrm{ILs}\left[\mathrm{PF}_{6}\right] \mathrm{Pd}^{0}$ (Pd equivalent $\left.10 \mu \mathrm{mol}\right), 35 \mathrm{mmol}$ nitrobenzene. } \\
\hline \multicolumn{9}{|c|}{ b $100 \mathrm{mg}$ Pd/PEG (Pd loading $3.75 \%$ ) and $1 \mathrm{mmol}$ nitrobenzene in $20 \mathrm{~mL}$ ethanol. } \\
\hline \multicolumn{9}{|c|}{ c $20 \mathrm{mg} \mathrm{Fe} \mathrm{O}_{4}-\mathrm{NH}_{2}-\mathrm{Pd}(\mathrm{Pd}$ loading $8.43 \mathrm{wt} \%)$ and $1 \mathrm{mmol}$ nitrobenzene in $5 \mathrm{~mL}$ EtOH. } \\
\hline \multicolumn{9}{|c|}{${ }^{\mathrm{d}} \mathrm{Pd} @ \mathrm{SiO}_{2}(\mathrm{Pd}$ equivalent $2.1 \mathrm{mg}), 2 \mathrm{mmol}$ nitrobenzene and $15 \mathrm{~mL}$ THF in a $30 \mathrm{~cm}^{3}$ autoclave. } \\
\hline
\end{tabular}

\section{References}

(1) Li, J.; Shi, X.-Y.; Bi, Y.-Y.; Wei, J.-F.; Chen, Z.-G. Pd Nanoparticles in Ionic Liquid Brush: A Highly Active and Reusable Heterogeneous Catalytic Assembly for SolventFree or On-Water Hydrogenation of Nitroarene under Mild Conditions. ACS catal. 2011, 1, 657-664.

(2) Harraz, F. A.; EI-Hout, S. E.; Killa, H. M.; Ibrahim I. A. Palladium Nanoparticles Stabilized by Polyethylene Glycol: Efficient, Recyclable Catalyst for Hydrogenation of Styrene and Nitrobenzene. J. Catal. 2012, 286, 184-192.

(3) Zhang, F. W.; Jin, J.; Zhong, X.; Li, S. W.; Niu, J. R.; Li, R.; Ma, J. T. Pd Immobilized on Amine-Functionalized Magnetite Nanoparticles: a Novel and Highly Active Catalyst for Hydrogenation and Heck Reactions. Green Chem. 2011, 13, 1238-1243.

(4) Tamura, M.; Kon, K.; Satsuma, A.; Shimizu, K. Volcano-Curves for Dehydrogenation of 2-Propanol and Hydrogenation of Nitrobenzene by $\mathrm{SiO}_{2}$-Supported Metal Nanoparticles Catalysts As Described in Terms of a d-Band Model. ACS Catal. 2012, 2, 1904-1909. 